\title{
Thermal States of Anyonic Systems
}

\author{
S. Iblisdir ${ }^{1}$, D. Pérez-García ${ }^{2}$, M. Aguado ${ }^{3}$, J. Pachos $^{4}$ \\ ${ }^{1}$ Dpt. Estructura i Constituents de la Materia, \\ Universitat Barcelona, 08028 Barcelona, Spain \\ ${ }^{2}$ Dpt. Análisis Matemático, \\ Universitad Complutense de Madrid, 28040 Madrid, Spain \\ ${ }^{3}$ Max Planck Institut für Quantenoptik, \\ Garching D-85748, Germany \\ ${ }^{4}$ School of Physics and Astronomy, \\ University of Leeds, Leeds LS2 9JT, United Kingdom
}

\begin{abstract}
A study of the thermal properties of two-dimensional topological lattice models is presented. This work is relevant to assess the usefulness of these systems as a quantum memory. For our purposes, we use the topological mutual information $I_{\text {topo }}$ as a "topological order parameter". For Abelian models, we show how $I_{\text {topo }}$ depends on the thermal topological charge probability distribution. More generally, we present a conjecture that $I_{\text {topo }}$ can (asymptotically) be written as a Kullback-Leitner distance between this probability distribution and that induced by the quantum dimensions of the model at hand. We also explain why $I_{\text {topo }}$ is more suitable for our purposes than the more familiar entanglement entropy $S_{\text {topo }}$. A scaling law, encoding the interplay of volume and temperature effects, as well as different limit procedures, are derived in detail. A non-Abelian model is next analysed and similar results are found. Finally, we also consider, in the case of a one-plaquette toric code, an environment model giving rise to a simulation of thermal effects in time.
\end{abstract}

\section{Introduction}

Quantum mechanics has significantly shaped our current understanding of condensed matter systems. It has proven to be deeply insightful when aiming to explain, say, transport of charged (quasi-) particles in semiconductors, magnetism in metallic alloys or cohesive properties of solids [1. But when it comes to macroscopic systems that exhibit a highly non-classical behavior, many fundamental issues are still poorly understood. An example of such systems is a fractional quantum Hall effect sample (FQHE), i.e. a specific two-dimensional electron gas subject to a strong perpendicular magnetic field. There the trans- 
verse conductivity appears in plateaux at fractional values of the filling factor, much in contrast to what one would expect classically [2]. Besides their fundamental interest, the exotic phases exhibited by these systems are important in that they may lead to new technological applications. Indeed, they could allow for intrinsically fault-tolerant quantum computation [3, 4. These phases are not separated by a symmetry whose absence or presence can be detected by a local order parameter. Rather, it has been realized that they are associated with topological order.

In the effort towards understanding this notion, an important contribution has been the research towards lattice spin models. Certain such systems are exactly solvable [3, 5, 6, 7], in the sense that their low energy sectors can be analytically determined. Although the corresponding interactions are local and frustration-free, they possess the essential properties of topologically ordered systems: (i) when the system is defined on a surface with non-trivial topology, the ground state has a non-local degeneracy that cannot be detected locally, (ii) excitations have exotic statistics. These features turn these models into an interesting alternative to FQHE systems, when it comes to study topological order. Initially, topological spin lattice systems were proposed as a reliable quantum memory [3, 8, 9] and some non-Abelian versions were shown to allow for universal fault-tolerant quantum computation [10. Quantum information would be encoded in non-local degrees of freedom of such systems and would therefore be immune to local perturbations. Fault-tolerant quantum computation could be performed by creating excitations (initialization), braiding them (unitary evolution), and fusing them back together (read-out) [4, 11.

In this work, which is partly an extension of [12], we focus on an entropic order parameter, the topological mutual information, $I_{\text {topo }}$, and use it to study how topological matter behaves in the presence of temperature. For concreteness, we restrict our analysis to two paradigmatic models: the toric code and the $D\left(S_{3}\right)$ "superconductor" [3, 13. We will see that at any fixed finite temperature, $I_{\text {topo }}$ is non-zero only when the size of the system is finite. Next, for fixed finite system size, there is always a temperature regime where the order parameter assumes a constant value before dropping to zero. A similar behavior is observed when the size of the system is increased for a fixed value of the temperature. Importantly, we exhibit a scaling relation that tells how much the temperature should be decreased to compensate for an increase of the size of the system, if the system is to remain topologically ordered. The exact behaviour of topological ordered systems with respect to temperature has received much attention recently. From a static point of view, the first results indicating the fragility of the $2 \mathrm{D}$ toric code against temperature can be found in [14, 15]. More recently, the authors of [16, 17] have shown the fragility of this system during the thermalization dynamics. In [18, 19] a more general approach is taken to show the impossibility of having a self correcting quantum memory if one restricts the search to 2D stabilizer codes. Apart from providing exact scaling relations, our work is the first to deal with the non-Abelian situation. A different new approach in this respect can be found in [20]. Though $I_{\text {topo }}$ is initially introduced 
as the natural generalization of the topological entropy $S_{\text {topo }}$ of Kitaev-Preskill 21] and Levin-Wen 22] to the thermal situation, the usefulness of this quantity will become clear throughout the paper. In particular, at least for the toric code, we explicitly relate $I_{\text {topo }}$ with the probability distribution of topological sectors in a region, connecting in this way the value of $I_{\text {topo }}$ with the ability of a system to perform quantum computing tasks at finite temperature. Moreover, we show how $I_{\text {topo }}$ allows to distinguish between quantum double models which share the same value of $S_{\text {topo }}$ at zero temperature.

The paper is structured as follows. In Section 2, we introduce $I_{\text {topo }}$ and discuss some of its properties. In Section 3 , we compute $I_{\text {topo }}$ for the toric code and show explicitly its dependence on size and temperature. We also explain how to simulate the behavior of $I_{\text {topo }}$ in a one-paquette toric code with current technology. In Section 4 the behavior of $I_{\text {topo }}$ for the non-Abelian case is studied. The general formalism is presented and the special case of $D\left(S_{3}\right)$ is given in detail. We provide numerical evidence of the fact that $I_{\text {topo }}$ depends on size and temperature exactly as for the toric code. Finally, in Section 5 we discuss the conclusions and implications of our work.

\section{Topological mutual information}

Our analysis is based on the idea that constant corrections to area laws are typical signatures of topological order. Let us have a closer look at this property. Consider a bipartition $R: R^{c}$ of a given system in a pure state of its ground subspace, and assume that the von Neumann entropy of the reduced density operator of $R, S_{R}=-\operatorname{tr} \rho_{R} \ln \rho_{R}$ satisfies:

$$
S_{R}=\alpha^{\prime}|\partial R|-\gamma^{\prime}+\epsilon(|\partial R|),
$$

where $\alpha^{\prime}$ is a constant, $|\partial R|$ denotes the size of the boundary of $R$ and where $\epsilon$ tends to zero when $|\partial R|$ tends to infinity. As discussed in 23, 22, 21, systems with a non-zero value of the topological entropy, $\gamma^{\prime}$, are topologically ordered. Indeed, $\gamma^{\prime}$ is related to the total quantum dimension $\mathcal{D}$ of the anyonic model describing the excitations:

$$
\gamma^{\prime}=\ln \mathcal{D}=\ln \sqrt{\sum_{q} d_{q}^{2}},
$$

where $d_{q}$ is the quantum dimension associated with anyon type $q$ [22, 21]. The trivial case with $\mathcal{D}=1$, i.e. $\gamma^{\prime}=0$, corresponds to non-topological models, where the only contribution to $\mathcal{D}$ comes from the vacuum.

Let us consider a system defined on a closed surface $\Sigma$. In the remainder of this paper, $\Sigma$ will either be a torus or a sphere. It was shown in 22,21 that $\gamma^{\prime}$ can be expressed as a linear combination of entropies of regions of $\Sigma$. For example, if $\Sigma$ is divided into four regions, as indicated in Fig. 1. then 21.

$$
\gamma^{\prime}=S_{A}+S_{B}+S_{C}-S_{A B}-S_{A C}-S_{B C}+S_{A B C} .
$$




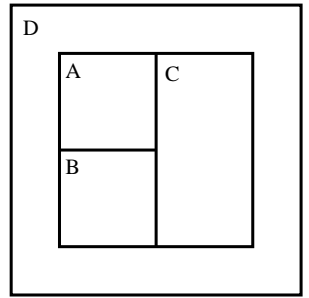

Figure 1: Division of a torus or a sphere into four regions.

Actually, there is much freedom in constructing linear combinations of entropies that are topologically invariant, i.e. invariant under local deformations of the boundaries. It is reasonable to require that the regions $A, B, C$ be treated on the same footing, and thus write such a linear combination as $I_{\text {topo }}=a^{1}\left(S_{A}+\right.$ $\left.S_{B}+S_{C}\right)+a^{2}\left(S_{A B}+S_{A C}+S_{B C}\right)+a^{3} S_{A B C}+b^{1}\left(S_{A D}+S_{B D}+S_{C D}\right)+b^{2}\left(S_{A B D}+\right.$ $\left.S_{A C D}+S_{B C D}\right)+b^{3} S_{A B C D}+x S_{D}$. Consider a deformation of the frontier between regions $C$ and $D$ away from any triple point. Since the deformation is well inside the region $C D$, we have $\Delta S_{C D}=\Delta S_{A C D}=\Delta S_{B C D}=\Delta S_{A B C D}=0$. Since it is local between the regions $C$ and $D, \Delta S_{C}=\Delta S_{A C}=\Delta S_{B C}=\Delta S_{A B C}$ and $\Delta S_{D}=\Delta S_{A D}=\Delta S_{B D}=\Delta S_{A B D}$. Therefore

$$
\Delta I_{\text {topo }}=\left(a^{1}+2 a^{2}+a^{3}\right) \Delta S_{C}+\left(2 b^{1}+b^{2}+x\right) \Delta S_{D} .
$$

So, in order to get a topological invariant, we must have that $a^{1}+2 a^{2}+a^{3}=2 b^{1}+$ $b^{2}+x=0$. Now let us consider a triple point deformation, at the intersection between the regions $B, C$ and $D$, say. Reasoning as before, we get

$$
\begin{aligned}
& \Delta I_{\text {topo }}=\left(a^{1}+a^{2}\right)\left(\Delta S_{B}+\Delta S_{C}\right)+\left(a^{2}+a^{3}\right) \Delta S_{B C} \\
& +\left(b^{1}+b^{2}\right)\left(\Delta S_{B D}+\Delta S_{C D}\right)+\left(b^{1}+x\right) \Delta S_{D} .
\end{aligned}
$$

We thus get stronger conditions: $a^{1}=-a^{2}=a^{3}$ and $b^{1}=-b^{2}=-x$. The entropy of the total system $S_{A B C D}$ is irrelevant, as expected; it is invariant under boundary deformations. Hence, there is no constraint on $b^{3}$. For $a^{1}=$ $1, b^{1}=b^{3}=x=0$, we recover the topological entropy, $\gamma^{\prime}$, defined in [21. In the following, we work with the choice $a^{1}=-b^{1}=-b^{3}=x=1$. This choice yields

$$
I_{\mathrm{topo}}=I_{A}+I_{B}+I_{C}-I_{A B}-I_{A C}-I_{B C}+I_{A B C},
$$

which amounts to replace the von Neumann entropies appearing in the definition of the topological entropy 21] by quantum mutual information. $\left(I_{R}\right.$ is defined as $S_{R}+S_{R_{c}}-S_{R \cup R_{c}}$.)

At finite temperature, the von Neumann entropy of a region $R, S_{R}$, is not a measure of correlations between $R$ and the rest of the system, as it is in the pure 
state case. In contrast, the quantum mutual information, $I_{R}$, still is. Moreover, $S_{R}$ does not obey an area law of the form (1) anymore, whereas for the lattice systems we are going to study, the mutual information still does 11 . That is, the properties of the von Neumann entropy that make $\gamma^{\prime}$ a topological order parameter at zero temperature are no longer valid at finite temperature. But they still hold for the quantum mutual information. This is why we choose to work here with $I_{\text {topo }}=\gamma$ instead of $\gamma^{\prime}$. In Section 3.5. we will further discuss the behaviour of $\gamma^{\prime}$.

We close this section by showing that any linear combination of entropies that is topologically invariant should, in general, involve a division of the surface $\Sigma$ into at least four regions. It will be enough to consider the case where the whole system is in a pure state. We would like to construct a particular linear combination of entropies that isolates $\gamma$ from the area part. It is clear that partitioning the surface into a region $R$ and its complement $R^{c}$ cannot provide such a quantity. Indeed, in this case $S_{R}=S_{R_{c}}, S_{R \cup R_{c}}=0$ and $\partial R$ and $|\gamma|$ have a common fate. Three regions $A, B$ and $C$ are not sufficient neither. This is easily shown considering a system in a pure state. Let $l_{A}, l_{B}$ and $l_{A B}$ denote respectively the length of the boundary between region $A$ and region $C$, region $B$ and region $C$, and region $A$ and region $B$, and let $n_{A}$ denote the number of connected pieces that make region $A, n_{B}$ and $n_{A B}$ are defined likewise. From the relations $S_{A}+S_{B}=\alpha\left(l_{A}+l_{B}+l_{A B}\right)-\left(n_{A}+n_{B}\right) \gamma$, $S_{A}-S_{B}=\alpha\left(l_{A}-l_{B}\right)+\left(n_{A}-n_{B}\right) \gamma$ and $S_{A B}=S_{C}=\alpha\left(l_{A}+l_{B}\right)-n_{A B} \gamma$, we see that it is impossible to construct a linear combination of entropies that cancels all boundary contributions and leaves only the topological contributions. Thus, four is the minimal number of pieces required in order to partition $\Sigma$ in such a way that it gives a boundary-independent quantity in a non-trivial way.

\section{The toric code}

The toric code is a simple topological model, with a Hamiltonian that can be diagonalized exactly [3]. It is called a 'code' because it is a quantum error correcting code; two logical qubits are encoded in the physical system. An optical implementation of a four-qubit toric code is possible since its ground state is a GHZ state [25], and proposals to create the ground state of large toric codes, as well as elementary excitations in optical lattices are described in [26. In this section, we study how $I_{\text {topo }}$ behaves as the (inverse) temperature, $\beta$, and the size of the system, $L$, are varied. A similar calculation has been presented in [15. We nevertheless present our alternative approach in details because it differs in two important respects. First, it is $\gamma^{\prime}$ which is used in [15] as a topological order parameter. Second, the simplicity of our alternative analysis has allowed us to get analytically more general as well as new results such as the scaling laws discussed in Section 3.3 . Moreover, this calculation

\footnotetext{
${ }^{1}$ Note that under quite general assumptions, a weak form of area law always holds at finite temperature: $I_{R} \leq \alpha|\partial R|$, for some constant $\alpha$ [24].
} 
helps understand better how to compute the topological mutual information for non-Abelian models.

\subsection{Spectrum}

Let us consider a torus tiled into $L \times L$ square plaquettes and associate a twolevel system (qubit) with each edge of the obtained lattice. We assume that these qubits interact through the Hamiltonian

$$
H=-J \sum_{p} B_{p}-J^{\prime} \sum_{s} A_{s},
$$

where the index $p$ (resp. $s$ ) runs over all plaquettes (resp. vertices) of the tiling. The operator $B_{p}$ involves all the spins surrounding the plaquette $p$, while the operator $A_{s}$ involves all the spins with one end at $s$. They are defined as follows:

$$
B_{p}=\prod_{i \in p} \sigma_{i}^{z}, \quad A_{s}=\prod_{i \in s} \sigma_{i}^{x},
$$

and represented on Fig.2 The coupling constants $J$ and $J^{\prime}$ will be chosen to be both equal to 1 , for the sake of simplicity. But all our analysis can be straightforwardly generalised to arbitrary values of $J$ and $J^{\prime}$.
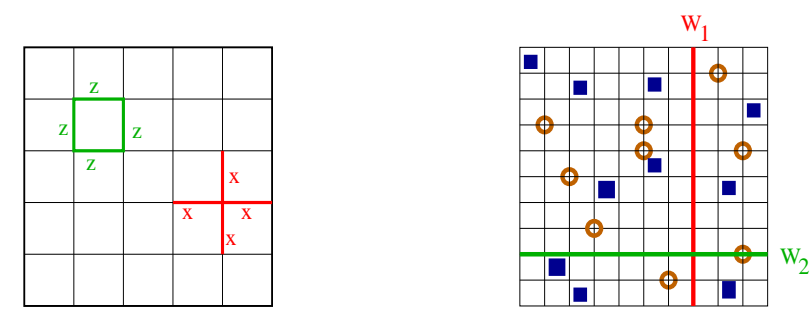

Figure 2: (Left) Pictural representation of the $B_{p}$ and the $A_{s}$ operators. (Right) An eigenstate of the toric code lattice. Elementary excitations can be either of flux type, depicted by squares in plaquettes, or of charge type depicted by hollow dots on vertices. The strings along the torus correspond to Wilson loops.

Due to the topology of the torus, we have that

$$
\Pi_{p} B_{p}=\Pi_{s} A_{s}=1 .
$$

This constraint means that the number of excited plaquettes (resp. excited vertices) is always even. The Hamiltonian $H$ is a sum of local terms all commuting with each other. We observe that although this property facilitates the diagonalization of $H$, it is not sufficient to guarantee that it is easy to solve. What makes $H$ exactly diagonalizable is that, as it turns out, its eigenstates of $H$ 
can have arbitrary eigenvalues of the operators $B_{p}$ and $A_{s}$, up to the constraint (9). Therefore, each eigenstate of $H$ is given by a triple of "quantum numbers": $|\phi, c, w\rangle$, see Fig. 2 A pattern $\phi$ denotes the position of all plaquette or "fluxtype" excitations. Another pattern, $c$, indicates the position of all vertex or "charge-type" excitations. Finally, $w$ indexes the degeneracy of the state for a fixed configuration of defects. This quantum number is made of two bits, $w_{1}$ and $w_{2}$, that label the values of the integrals of motion of $z$-operators around non-contractible loops on the torus $\underline{3}$. (Wilson loops). We have

$$
H|\phi, c, w\rangle=\left(E_{0}+2|\phi|+2|c|\right)|\phi, c, w\rangle,
$$

where $E_{0}=-2 L^{2}$ is the ground state energy and $|\phi|$ (resp. $|c|$ ) denotes the number of flux excitations (resp. charge excitations) of the pattern $\phi$ (resp. $c$ ). The eigenvalues of $H$ satisfy $E_{n}-E_{n+1}=-4$ and range between $-2 L^{2}$ and $2 L^{2}$. Also, if $P_{i}$ denotes the projector onto the sector of energy $E_{i}=E_{0}+4 i$ and $d_{i}=\operatorname{tr} P_{i}$ denotes its dimension, we have that

$$
d_{i}=4 \sum_{n_{\phi}, n_{c} \leq L^{2} / 2} \sum_{n_{\phi}+n_{c}=i}\left(\begin{array}{c}
L^{2} \\
2 n_{\phi}
\end{array}\right)\left(\begin{array}{c}
L^{2} \\
2 n_{c}
\end{array}\right) .
$$

One can check that $\sum_{i} d_{i}=2^{2 L^{2}}$.

\section{2 von Neumann entropy}

In the following, we shall consider a situation in which the system is immersed in a bath at inverse temperature $\beta$ and is let to thermalize. Since $H=\sum_{i=0}^{L^{2}} E_{i} P_{i}$, the partition function of this model reads

$$
Z(\beta, L)=\operatorname{tr} e^{-\beta H}=\sum_{i=0}^{L^{2}} e^{-\beta E_{i}} d_{i},
$$

This series can be easily summed up (see [14] or details in Appendix A). We get

$$
Z(\beta, L)=\left((2 \cosh \beta)^{L^{2}}+(2 \sinh \beta)^{L^{2}}\right)^{2} .
$$

The thermal state of Eq. (7) reads

$$
\rho_{\mathrm{th}}=e^{-\beta H} / Z(\beta, L) \text {. }
$$

The von Neumann entropy, $S_{\mathrm{tot}}=-\operatorname{tr} \rho_{\mathrm{th}} \ln \rho_{\mathrm{th}}$, of the whole torus is then easily derived from the partition function thanks to the identity

$$
S_{\mathrm{tot}}=-\frac{\beta}{Z(\beta, L)} \frac{\partial}{\partial \beta} Z(\beta, L)+\ln Z(\beta, L) .
$$

We now compute the von Neumann entropy of a connected region $R \subset \Sigma$, $S_{R}$. A couple of observations about the reduced state, $\rho_{R}$, allows to get an 
analytic expression for $S_{R}$. First, we consider a fixed eigenstate $|\phi, c, w\rangle$ of the Hamiltonian (7). The reduced state $\rho_{R}(\phi, c, w)=\operatorname{tr}_{R_{c}}|\phi, c, w\rangle\langle\phi, c, w|$ does not depend on $w$ if $R$ is contractible; homologically non-trivial loops are necessary to measure $w$. Also, two states $(\phi, c, w) \neq\left(\phi^{\prime}, c^{\prime}, w^{\prime}\right)$ are orthogonal whenever $\left(\phi_{R}, c_{R}\right) \neq\left(\phi_{R}^{\prime}, c_{R}^{\prime}\right)$ since they can be discriminated by measuring $A_{s}$ operators or $B_{p}$ operators having support on $R$. Next, it is useful to distinguish three kinds of plaquette excitations: those with support fully on $R, \phi_{R}$, those with support fully on $R_{c}, \phi_{R_{c}}$, and the others, $\phi_{\partial R}$. Similarly, we divide vertex excitations into three kinds: $c_{R}, c_{R_{c}}, c_{\partial R}$. In order to lighten the notations, we use the symbol $\mathbf{q}$ to label configurations of defects, both plaquette and vertex, i.e. $\mathbf{q} \equiv(\phi, c)$. Crucially, excitations of the $\partial R$ type can be driven inside $R_{c}$ by application of Pauli operators acting on links of $R_{c}$, that is:

$$
\left|\mathbf{q}_{R}, \mathbf{q}_{R_{c}}, \mathbf{q}_{\partial R}, w\right\rangle=U_{R_{c}}^{\prime \prime}\left|\mathbf{q}_{R}, \mathbf{q}_{R_{c}}^{\prime}, w\right\rangle,
$$

for some unitary operator $U_{R_{c}}^{\prime \prime}$ and some configuration of defects in $R_{c}, \mathbf{q}_{R_{c}}^{\prime}$. This property is illustrated in Fig. 3

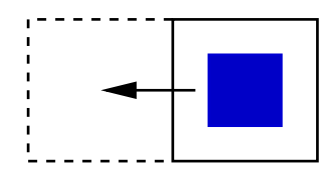

Figure 3: Solid edges belong to a region $R$, while dashed edges belong to $R_{c}=$ $\Sigma \backslash R$. A plaquette excitation on the right $\partial R$ plaquette can be driven inside $R_{c}$ applying $\sigma^{x}$ on the edge separating $R$ from $R_{c}$.

All excitations inside $R$ can be fused into a single excitation $q_{R}^{1}$ located on a site 2 , using a unitary whose support is fully in $R$ and similarly for $R_{c}$. In turn, the state $\left|q_{R}^{1}, q_{R_{c}}^{1}, w\right\rangle$ can be created from a ground state $|\xi, w\rangle$ by application of Pauli operators along strings connecting the site where $q_{R}^{1}$ is located to the site where $q_{R_{c}}^{1}$ is located. So,

$$
|\mathbf{q}, w\rangle=U_{R}\left(\mathbf{q}_{R}\right) \otimes U_{R_{c}}\left(\mathbf{q}_{R_{c}}, \mathbf{q}_{\partial R}\right)|\xi, w\rangle
$$

for some unitary operators $U_{R}\left(\mathbf{q}_{R}\right)$ and $U_{R_{c}}\left(\mathbf{q}_{R_{c}}, \mathbf{q}_{\partial R}\right)$. We are now in a position to characterise $\rho_{R}$ and compute $S_{R}$. The thermal state of the toric code can be written explicitly as

$$
\rho_{\mathrm{th}}=\sum_{w, \mathbf{q}} \frac{e^{-\beta\left(E_{0}+\Delta E|\mathbf{q}|\right)}}{Z(\beta, L)}|\mathbf{q}, w\rangle\langle\mathbf{q}, w|,
$$

where $\Delta E=2$ is the energy associated with a single excitation (plaquette or vertex). Therefore, for a non-contractible region $R$,

$$
\rho_{R}=\sum_{w, \mathbf{q}_{R}} C\left(q_{R}\right) \operatorname{tr}_{R_{c}}\left[U_{R}\left(\mathbf{q}_{R}\right)|\xi, w\rangle\langle\xi, w| U_{R}\left(\mathbf{q}_{R}\right)^{\dagger}\right],
$$

\footnotetext{
${ }^{2} \mathrm{As}$ in [3], we call a site a combination of a vertex and an adjacent plaquette.
} 
where

$$
C\left(\mathbf{q}_{R}\right)=\sum_{\mathbf{q}_{R_{c}}, q_{\partial R}} e^{-\beta\left(E_{0}+2\left|\mathbf{q}_{R}\right|+2\left|\mathbf{q}_{R_{c}}\right|+2\left|\mathbf{q}_{\partial R}\right|\right)} / Z(\beta, L) .
$$

Note that $4 C\left(\mathbf{q}_{R}\right)$ is the marginal probability of a configuration of defects $\mathbf{q}_{R}$. The decomposition (19) allows to compute $S_{R}$. Indeed, from the identity

$$
S\left(\bigoplus_{i} \lambda_{i} \rho_{i}\right)=-\sum_{i} \lambda_{i} \ln \lambda_{i}+\sum_{i} \lambda_{i} S\left(\rho_{i}\right)
$$

we find that

$$
S_{R}=S_{R}^{\mathrm{gs}}-\sum_{\mathbf{q}_{R}} 4 C\left(\mathbf{q}_{R}\right) \ln \left(4 C\left(\mathbf{q}_{R}\right)\right),
$$

where $S_{R}^{\mathrm{gs}}$ is the von Neumann entropy of the region $R$ when the system is in a pure ground state $|\xi, w\rangle: S_{R}^{\mathrm{gs}}=(|\partial R|-1) \ln 2[23]$.

It turns out that the sums appearing in Eq. 21) can be carried out exactly (see details in Appendix A). The result is that the entropy of a region can be expressed as

$$
S_{R}=S_{R}^{\mathrm{gs}}+V\left(\beta, N_{p}(R), L\right)+V\left(\beta, N_{*}(R), L\right),
$$

with

$$
\begin{aligned}
& V(\beta, N, L)=N \ln \left(1+e^{-2 \beta}\right)+\ln \left(1+\theta^{L^{2}}\right)+\frac{N \beta e^{-\beta}}{\cosh \beta} \frac{1-\theta^{L^{2}-1}}{1+\theta^{L^{2}}}+\ln 2 \\
& -\frac{\left(1+\theta^{N}\right)\left(1+\theta^{L^{2}-N}\right)}{2\left(1+\theta^{L^{2}}\right)} \ln \left(1+\theta^{L^{2}-N}\right) \\
& -\frac{\left(1-\theta^{N}\right)\left(1-\theta^{L^{2}-N}\right)}{2\left(1+\theta^{L^{2}}\right)} \ln \left(1-\theta^{L^{2}-N}\right),
\end{aligned}
$$

where $\theta=\tanh \beta$.

As we can see, the entropy of a region separates neatly into a pure state contribution and a finite temperature contribution. The first only involves the area of the region, while the second depends on its volume $\left(N_{p}(R)\right.$ and $\left.N_{*}(R)\right)$. By increasing the temperature, we pass from an area law to a volume law, as expected. The calculations are almost identical when $R$ is only semi-contractible. The only difference is that $\rho_{R}$ will depend on only one of the Wilson loops, and the entanglement entropy picks a $-\ln 2$ additive correction. When $R$ is completely contractible, the correction is $-\ln 4$. 


\subsection{Dependence of $I_{\text {topo }}$ on size and temperature}

Using Eq. (23), we have plotted the topological mutual information (6) for tori of various sizes, see Fig. 4. For a fixed torus size, we observe that there is a region of values of $\beta$ such that $I_{\text {topo }}$ is non-zero and stationary. Then, as the temperature is increased, $I_{\text {topo }}$ smoothly vanishes. Interestingly, the transition does not become more abrupt when the size of the system is increased. Rather, the curves displayed are similar.

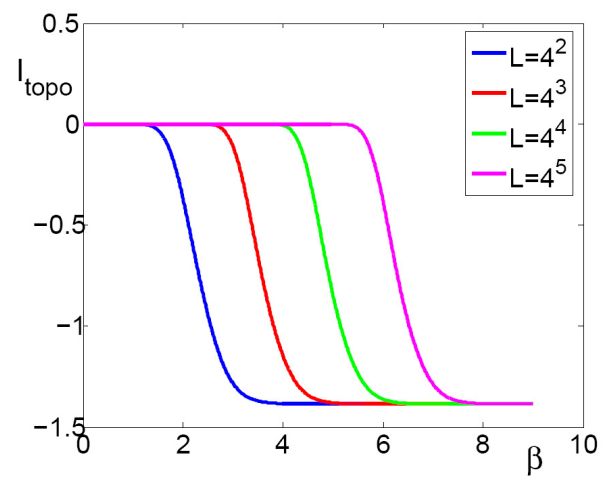

Figure 4: Topological mutual information as a function of $\beta$ for tori of size $4^{2} \times 4^{2}, 4^{3} \times 4^{3}, 4^{4} \times 4^{4}$ and $4^{5} \times 4^{5}$. The size of the region $\mathrm{A}$, as indicated in Fig. 1 is $k \times k=(L / 4) \times(L / 4)$.

From Fig. 4, we also see that, when the system size increases, the temperature at which the transition occurs decreases. Actually, in the limit of large codes, the transition temperature vanishes. When systems described by a local hamiltonian are left in thermal equilibrium, their mutual information is bounded by a constant times their area 24]. As it turns out, the systems we are studying obey a strict area law, i. e. the quantum mutual information between a region $R$ and the rest of the sytem satisfies:

$$
I_{R}=\alpha(\beta, L)|\partial R|-\gamma(\beta, R, L) .
$$

For the toric code, we can compute

$$
\alpha_{\infty}(\beta)=\lim _{|\partial R| \rightarrow \infty} I_{R} /|\partial R|, \quad \gamma_{\infty}(\beta)=\lim _{|\partial R| \rightarrow \infty}\left(I_{R}-\alpha_{\infty}(\beta)|\partial R|\right) .
$$

Interestingly, we find slightly different results for $\gamma_{\infty}(\beta)$, depending on how this limit is taken. One possibility is to first consider the limit for $L \rightarrow \infty$, and then let the size of the region $R$ grow. One finds:

$$
\begin{aligned}
& \gamma(\beta, R, \infty)=2 \ln 2 \\
& +\frac{1+\theta^{\bar{N}_{*}(R)}}{2} \ln \frac{1+\theta^{\bar{N}_{*}(R)}}{2}+\frac{1-\theta^{\bar{N}_{*}(R)}}{2} \ln \frac{1-\theta^{\bar{N}_{*}(R)}}{2}
\end{aligned}
$$




$$
+\frac{1+\theta^{\bar{N}_{p}(R)}}{2} \ln \frac{1+\theta^{\bar{N}_{p}(R)}}{2}+\frac{1-\theta^{\bar{N}_{p}(R)}}{2} \ln \frac{1-\theta^{\bar{N}_{p}(R)}}{2},
$$

where $\theta=\tanh \beta$, and where $\bar{N}_{p}(R)=L^{2}-N_{p}\left(R_{c}\right)$ denotes the number of plaquettes within a region $R$ and at its border. $\bar{N}_{*}(R)$ is defined likewise.

Eq. 25 lends itself to a simple interpretation. Let $p_{e}$ denote the probability that a plaquette or a site is excited. The mean energy of the system reads $\langle H\rangle=E_{0}+4 p_{e} L^{2}$. From $\langle H\rangle Z(\beta, L)=-\partial Z(\beta, L) / \partial \beta$, we find that in the limit $L \rightarrow \infty, p_{e}=(1-\theta) / 2$. On another hand, ignoring total anyonic charge conservation, the probability that a region $R$ containing $N_{p}(R)$ plaquettes has an even number of excited plaquettes reads

$$
p_{p}^{\text {even }}(R)=\frac{1}{2} \sum_{i=0}^{N_{p}(R)}\left(\begin{array}{c}
N_{p}(R) \\
i
\end{array}\right)\left(p_{e}^{i}+\left(-p_{e}\right)^{i}\right)\left(1-p_{e}\right)^{N_{p}(R)-i}=\frac{1}{2}\left(1+\theta^{N_{p}(R)}\right) .
$$

One can similarly calculate $p_{*}^{\text {even }}(R)$, the probability that the region $R$ contains an even number of excited vertices. One find the same expression with $N_{p}(R)$ replaced by $N_{*}(R)$. So the asymptotic limit of the topological mutual information can be rewritten as

$$
\gamma_{\infty}(\beta)=2 \ln 2-h_{2}\left(p_{p}^{\text {even }}(R)\right)-h_{2}\left(p_{*}^{\text {even }}(R)\right),
$$

where $h_{2}(x)=-x \ln x-(1-x) \ln (1-x)$ is the Shannon entropy of a binary outcome probability distribution, or equally simply as

$$
\gamma_{\infty}(\beta)=-D\left(\left\{p_{R}(q)\right\} \|\left\{p_{u}(q)\right\}\right),
$$

where $\left\{p_{u}\right\}$ is the four-event uniform probability distribution, $\left\{p_{R}\right\}$ denotes the thermal probability distribution associated with all possible values for the total anyonic charge: total plaquette flux in $R$ trivial and total electric charge in $R$ trivial, etc, and where $D\left(\left\{p^{1}\right\} \|\left\{p^{2}\right\}\right)=-\sum_{j} p_{j}^{1} \ln \left(p_{j}^{1} / p_{j}^{2}\right)$ denotes the KullbackLeitner pseudo-distance between two probability distributions $\left\{p^{1}\right\}$ and $\left\{p^{2}\right\}$ [27. We can see that $\lim _{k \rightarrow \infty} \gamma_{\infty}(\beta)=0$. We conjecture that the formula (28) is valid in general, with the probability distribution induced by the quantum dimensions:

$$
p_{u}(q)=\frac{d_{q}^{2}}{\mathcal{D}^{2}} .
$$

This relation has been verified for all Abelian quantum double models.

The other possibility, when studying the asymptotic behavior of the topological mutual information, is to let the size of the torus and the size of the region $R$, grow at the same rate. Let $\nu L \times \nu L$ denote the area of the region $R(\nu<1)$. In that case, keeping $\theta^{L^{2}}$ fixed is the only way to make the limit 
meaningful. Using the fact that $\lim _{L \rightarrow \infty} \theta^{L}=1$ in that case, one finds again Eq. 28), but the probabilities are slightly different now. For example

$$
p_{\text {even }}^{*}(R) \simeq \frac{\left(1+\theta^{\nu^{2} L^{2}}\right)\left(1+\theta^{\left(1-\nu^{2}\right) L^{2}}\right)}{2\left(1+\theta^{L^{2}}\right)}, \quad p_{\text {even }}^{p}(R) \simeq p_{\text {even }}^{*}(R) .
$$

These quantities are still the probabilities corresponding to the value of the total charge (flux) sector for region $R$ but subject to the global flux (charge) neutrality condition.

Eqs. 28 30) are very interesting in that they allow to extract a scaling law for the topological mutual information. In the simultaneous limit, i.e. for a fixed value of $\nu$, the topological mutual information only depends on the temperature

and size through the parameter $t=\tanh (\beta)^{L^{2}}$. In particular, a fixed value of $t$, and thus a fixed value of the topological mutual information, corresponds to the following relation between size and temperature

$$
\begin{aligned}
& \beta(t, L)=\ln L-\frac{1}{2} \ln \left(\frac{1}{2} \ln \frac{1}{t}\right)+O\left(L^{-2}\right), \\
& \frac{\partial T(t, L)}{\partial L}=\frac{-1}{L\left(\ln L-\frac{1}{2} \ln \left(\frac{1}{2} \ln \frac{1}{t}\right)+O\left(L^{-2}\right)\right)^{2}}+O\left(L^{-2}\right) .
\end{aligned}
$$

For a general Abelian quantum double, based on a group $G=\mathbb{Z}_{K_{1}} \times \ldots \times \mathbb{Z}_{K_{r}}$, these formulae generalise to

$$
\beta(t, L)=\ln L-\frac{1}{2} \ln \left(\frac{1}{K} \ln \frac{1}{t}\right)+O\left(L^{-2}\right),
$$

where $K=K_{1} \ldots K_{r}$, and where the scaling variable is now defined as

$$
t=\left(\frac{1-e^{-\beta \Delta E}}{1+(K-1) e^{-\beta \Delta E}}\right)^{L^{2}},
$$

where $\Delta E$ denotes again the energy associated with an excitation.

These relations tell us how an increase of the size of the system should be compensated by a decrease of temperature in order to maintain a fixed value of the topological mutual information. We believe that they constitute a qualitative nuance from the fact that at any fixed finite temperature, the topological mutual information, or the topological entropy, asymptotically vanishes when the size of the system is increased [15]. In particular, they show that the rate at which the temperature should be decreased, in order to maintain a fixed value of $\gamma$, decreases with the size of the system.

\subsection{Mean value of the $S$-matrix}

As we have seen, the sub-leading term in the area law for $I_{R: R^{c}}$ contains entropic information about the thermal probability distribution of topological sectors inside region $R$. In fact, we now wish to argue that this probability distribution is 
the fundamental quantity controlling in principle (up to implementation-specific problems) the usefulness of topological quantum memory and quantum computing by anyon braiding.

For concreteness we take the toric code model that has been initially proposed as topological memory [3]. Consider the process of creating a pair of electric and a pair of magnetic defects, braiding between one particle of each pair and subsequently annihilating both pairs, as shown in Fig. 5. The crucial property of these anyons is that the operation $U_{\mathrm{Hopf}}$ effecting this process on any state of the ground level multiplies it by -1 ; indeed, $U_{\mathrm{Hopf}}=-U_{R_{1}}^{\mathrm{e}} U_{R_{2}}^{\mathrm{m}}$, where $U_{R_{1}}^{\mathrm{e}}$ and $U_{R_{2}}^{\mathrm{m}}$ are the operators describing the processes associated with the electric and magnetic anyons separately, i.e., unlinked. Thus, the thermal

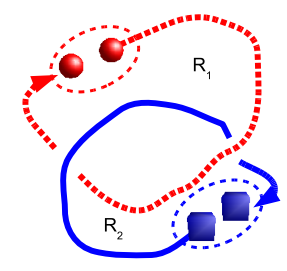

Figure 5: Braiding of anyons in the toric code. A pair of electric defects (spheres) and a pair of magnetic defects (cubes) are created. One electric and one magnetic defect are wound around each other, then both pairs are annihilated. The anyon trajectories define a two-dimensional projection of a Hopf link, whose components enclose regions $R_{1}$ and $R_{2}$.

expectation values are immediately related to (marginals of) the charge probability distributions since $U_{R_{1}}^{\mathrm{e}}$ and $U_{R_{2}}^{\mathrm{m}}$ measure the electric and magnetic charges inside their regions:

$$
\left\langle U_{\mathrm{Hopf}}\right\rangle_{\beta}=-\left\langle U_{R_{1}}^{\mathrm{e}}\right\rangle_{\beta}\left\langle U_{R_{2}}^{\mathrm{m}}\right\rangle_{\beta}=-\left(p_{\text {even }}^{*}\left(R_{1}\right)-p_{\text {odd }}^{*}\left(R_{1}\right)\right)\left(p_{\text {even }}^{p}\left(R_{2}\right)-p_{\text {odd }}^{p}\left(R_{2}\right)\right) .
$$

As a consequence, the expectation value of $U_{\mathrm{Hopf}}$ is controlled by scaling variables $\theta^{\operatorname{vol}\left(R_{1}\right)}$ and $\theta^{\operatorname{vol}\left(R_{2}\right)}$. We expect this will be the case for many anyonic models, where similar expectation values control the visibility of interferometry experiments 28. Note that Hopf-link-like processes define the elements of the topological $S$-matrix and twisted self-braiding of anyons yields their topological spin. Thus, these fundamental quantities of the anyon model are degraded at finite temperature at a rate controlled by the thermal charge probability distributions. The latter becomes the object that determines the appropriateness of the system to perform quantum computation at finite temperature.

In particular, $I^{\text {topo }}$ measures the Shannon entropy of this distribution. This is why we believe that it is a good topological order parameter. Note that in the ground level, the probability of finding anyons in a given region vanishes, therefore the probability of a given sector $q, p^{q}(R)$, becomes $\delta_{q, 1}$ and the distribution 
has zero Shannon entropy. For high temperatures the distribution approaches 29 , which is dictated only by the quantum dimensions of the anyons. Our conjecture 28 implies that this is the maximal Shannon entropy available to the distribution.

\subsection{Topological Entropy}

We now analyze the behavior of $\gamma^{\prime}$ introduced in Eq. (3). We start by observing that if one writes $\gamma^{\prime}$ as $\sum_{R} \sigma_{R} S_{R}$, where $R \in\{A, B, C, A B, A C, B C, A B C\}$ (see Fig. 11, then

$$
\sum_{R} \sigma_{R}\left(N_{p}(R)+N_{*}(R)\right)=1
$$

With this lattice relation and Eq. 22, one can see that in the limit where the sizes of all regions diverge,

$$
\gamma^{\prime} \rightarrow \ln 2-\frac{2 \beta}{e^{2 \beta}+1}-\ln \left(1+e^{-2 \beta}\right) .
$$

At fixed temperature, $\gamma^{\prime}$ does not vanish as the size of the system grows, a behavior that contrasts with that of $I_{\text {topo }}$. It actually becomes independent of the system size. Since, at finite temperature, the von Neumann entropy of a region is no longer a measure of its correlations with the rest of the system, it is not clear whether $\gamma^{\prime}$ actually still probes topological order. The discrepancy between the behaviour of $\gamma$ and that of $\gamma^{\prime}$ is made obvious here because magnetic and electric defects have been treated on an equal footing right from the start $\left(J=J^{\prime}\right.$ in Eq. (7)). Our results therefore do not contradict those of [15]). Note that $\gamma^{\prime}$ vanishes in the limit where $\beta$ tends to zero, as expected.

\subsection{The Case of One Plaquette}

In this subsection we develop a quantum simulation of a minimal toric code at finite temperature 25. We therefore consider a four qubit GHZ state, coupled to a single ancillary qubit, that plays the role of the environment. For a particular time-dependent coupling between the system and the environment, it is possible to reproduce the exact behavior of topological entanglement as a function of temperature, where the latter is now represented by time. This can be also viewed as a purification protocol of the topological thermal states. An experimental verification of this topological behavior could be demonstrated with state-of-the-art technology.

Consider a single plaquette of the toric code model [25]. The corresponding Hamiltonian can be given by

$$
H=-J \sigma_{1}^{x} \sigma_{2}^{x} \sigma_{3}^{x} \sigma_{4}^{x}-\sigma_{1}^{z} \sigma_{2}^{z}-\sigma_{2}^{z} \sigma_{3}^{z}-\sigma_{3}^{z} \sigma_{4}^{z}
$$

where we have omitted the term $\sigma_{4}^{z} \sigma_{1}^{z}$ as it is superfluous for generating a topologically ordered ground state. The corresponding ground state (vacuum) 
is the GHZ state

$$
|\xi\rangle=\left|G H Z_{4}\right\rangle=\frac{1}{\sqrt{2}}(|0000\rangle+|1111\rangle) .
$$

When the plaquette is occupied by an electric charge, the state of the system is $|e\rangle=\sigma_{1}^{z}|\xi\rangle=\frac{1}{\sqrt{2}}(|0000\rangle-|1111\rangle)$. It is possible to consider the thermal state of the system at temperature $T=1 / \beta$,

$$
\rho=\frac{e^{-H \beta}}{\operatorname{Tr}\left(e^{-H \beta}\right)} .
$$

We can calculate the topological mutual information for this system. Consider the splitting of the four qubits in the regions $A, B, C$ and $D$, as seen in Fig. 6 .

If we plot $I_{\text {topo }}$ as a function of the temperature for this system, we get Fig.7.
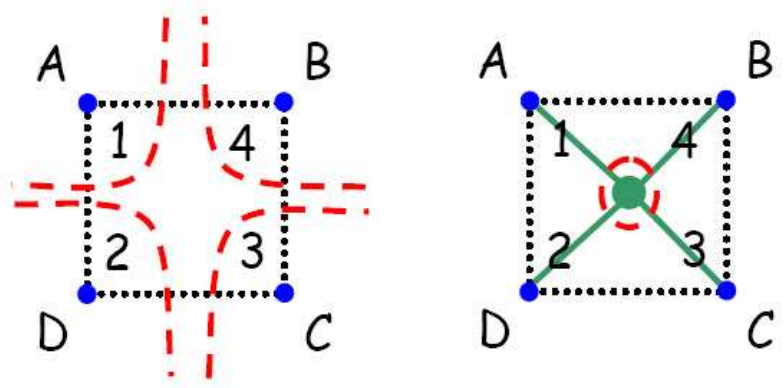

Figure 6: (Left) The one plaquette system of the toric code, which is in the GHZ state. Each subsystem $A, B, C$ and $D$ consists in a single qubit. (Right) The same system without the Hamiltonian (35) coupled to the environment, here taken to be one extra ancillary qubit. The latter can be coupled to all the plaquette qubits or equivalently to just one of them.

Since all the interaction terms in the Hamiltonian (35) commute with each other it is easy to evaluate the density matrix. For simplicity we focus on the case where $J \ll 1$ which reduces the density matrix to

$$
\rho_{\beta}=\frac{1}{2}\left(\mathbb{1}+\sigma_{1}^{x} \sigma_{2}^{x} \sigma_{3}^{x} \sigma_{4}^{x} \tanh \beta\right) .
$$

We would like to simulate the same behavior, but without the background Hamiltonian (35). We can reproduce the density matrix, $\rho_{\beta}$, with four noninteracting qubits initially in state $|\xi\rangle$ coupled to an environment. We take the environment to be an ancilla initially in state $\left|\psi_{a}\right\rangle=(|0\rangle+|1\rangle) / \sqrt{2}$ coupled to the system through the interaction

$$
H_{\mathrm{int}}=\omega \sigma_{1}^{z} \sigma_{a}^{z} .
$$




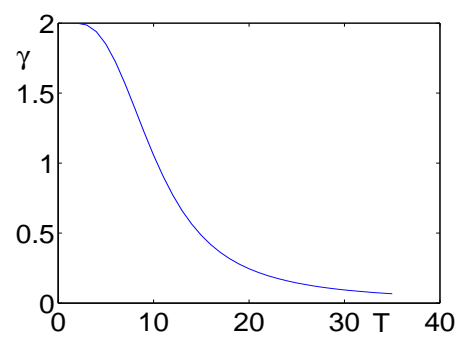

Figure 7: Topological mutual information, of the plaquette system as a function of the temperature, $T$. When the Hamiltonian (35) is present we observe a resilience of the topological character $(\gamma \sim 2)$ for small temperatures, which is lost for higher temperatures $(\gamma \sim 0)$.

Note that an interaction Hamiltonian that couples the ancilla symmetrically with all the qubits would give the same results. For $U(t)=\exp \left(-i H_{\text {int }} t\right)$ the time evolution due to (39) gives a reduced density matrix for the four qubits of the form

$$
\rho_{t}=\operatorname{tr}_{a}\left(U(t)\left|\xi, \psi_{a}\right\rangle\left\langle\xi, \psi_{a}\right| U^{\dagger}(t)\right) .
$$

For particular choices of a time dependent $\omega$, it is possible to make $\rho_{t}$ identical to $\rho_{\beta}$. Now, the time evolution of $\rho_{t}$ is identified with the increase in the temperature, $T$, of $\rho_{\beta}$. Indeed, if we choose the coupling as

$$
\omega(t)=\frac{J}{t^{2}} \cosh ^{-2} \frac{J}{t},
$$

then the temporal evolution of the system without the Hamiltonian (35) is equivalent to constantly increasing the temperature of the same system in the presence of the Hamiltonian.

Thus, the toric code plaquette with Hamiltonian 35 in a thermal state at finite temperature, $T$, has the same topological mutual information as a plaquette initially prepared in $|\xi\rangle$ and coupled to an ancilla in state $|+\rangle$, where time plays the role of temperature. By employing larger states and more ancillae, one could reproduce the finite temperature topological behavior of larger toric code systems.

\section{Non-Abelian models}

We now turn to a class of models featuring non-Abelian anyonic statistics [3]. We call these models "non-Abelian superconductors", as in 13 because of the braiding properties of their excitations. We mainly focus on a particular model based on the quantum double $D\left(S_{3}\right)$, because it is the simplest in the family 
we are considering. This model is paradigmatic though; our analysis can be straightforwardly generalized to models defined through the quantum double of any finite group. The $D\left(S_{3}\right)$ model is also important from a quantum information perspective because it allows to perform universal quantum computation [10. We start by briefly reviewing some properties of the hamiltonians we are considering. Then we show how the entropy of a region can be calculated. This result is employed to study the behavior of the topological mutual information at finite temperature.

\subsection{The $D\left(S_{3}\right)$ superconductor and its eight sectors}

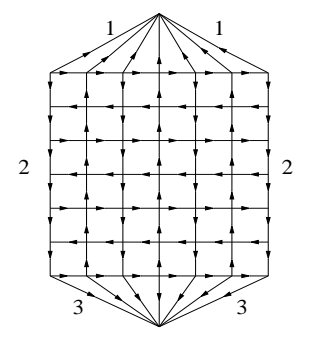

Figure 8: Tiled sphere. Equal numbers refer to identified sets of edges.

Consider an oriented lattice, $\Lambda$, on a sphere such as the one represented in Fig. 8. Let $G$ denote a finite group, where $e$ refers to its neutral element. With each edge of $\Lambda$, we associate a $|G|$-dimensional Hilbert space, $\mathcal{H}$, with an orthonormal 'computational' basis labelled by the group elements $\{|g\rangle: g \in G\}$. We now borrow a series of definitions introduced in [3]. We introduce the operators $L_{ \pm}$and $T_{ \pm}$, which act on the computational basis as $L_{+}(g)\left|g^{\prime}\right\rangle=\left|g g^{\prime}\right\rangle$, $T_{+}(g)\left|g^{\prime}\right\rangle=\delta_{g g^{\prime}}\left|g^{\prime}\right\rangle, L_{-}(g)\left|g^{\prime}\right\rangle=\left|g^{\prime} g^{-1}\right\rangle, T_{-}(g)\left|g^{\prime}\right\rangle=\delta_{g^{-1} g^{\prime}}\left|g^{\prime}\right\rangle$. We also define $L^{g}(j, s)=L_{-}(g)$ if $s$ is the origin of an edge $j$, whereas $L^{g}(j, s)=L_{+}(g)$ if $s$ is the endpoint of $j$. Also, if a plaquette $p$ is at the left (resp. right) of an edge $j$, we define $T_{g}(j, p)=T_{-}(g)$ (resp. $\left.T_{g}(j, p)=T_{+}(g)\right)$. We further define

$$
A_{g}(s)=\bigotimes_{j \in *(s)} L^{g}(j, s), \quad B_{g}(s, p)=\sum_{g_{1} \ldots g_{m}=g} \bigotimes_{m \in \square(p)} T_{g_{m}}\left(j_{m}, p\right),
$$

where in the definition of $B_{g}(s, p), j_{1}, \ldots, j_{m}$ are the boundary edges of $p$ listed in counterclockwise order, starting from and ending at some vertex $s$. The operators $A_{g}(s)$ and $B_{g}(s, p)$ commute when they share no link. Otherwise, they satisfy the following relations $[3]$ :

$$
A_{g_{1}} A_{g_{2}}=A_{g_{1} g_{2}}, \quad B_{g_{1}} B_{g_{2}}=\delta_{g_{1} g_{2}} B_{g_{1}}, \quad A_{g_{1}} B_{g_{2}}=B_{g_{1} g_{2} g_{1}^{-1}} A_{g_{1}}
$$

These commutation relations represent those of an algebra called the quantum double or Drinfeld algebra [29] that we denote as $D(G)$. 
From the operators

$$
A_{s}=\frac{1}{|G|} \sum_{g \in G} A_{g}(s), \quad \text { and } \quad B_{p}=B_{e}(p),
$$

it is possible to construct a Hamiltonian made of local mutually commuting terms, which has the same form as for the toric code, Eq. (7).

An elementary excitation of $H$ lives on a vertex and an adjacent plaquette that we call site. Each excitation is associated with a pair of data: an equivalence class of $G$ and a representation of the normalizer of an arbitrary element of this equivalence class [29, 3, 13. Each such pair of data corresponds to an irreducible representation of $D(G)$. Quasiparticles associated with the trivial equivalence class $\{e\}$ are called pure charges. Quasiparticles associated with trivial representations are called pure fluxes. The rest of the quasiparticles are called dyons. For the $D\left(S_{3}\right)$ model, the quasiparticles come in eight varieties that we label $A, \ldots, H[13$. One can construct operators that project the links related to a site onto a definite quasiparticle state. This is done upon observing that the operators $B_{h}(s, p) A_{g}(s)$ form a reducible representation of the quantum double $D\left(S_{3}\right)$. Each definite quasiparticle state corresponds to an irreducible representation of $D\left(S_{3}\right)$ contained in this reducible representation. Therefore, the projectors onto a given quasiparticle type can be constructed using characters of the corresponding irreducible representation [30, 31]:

$$
P_{q}=\sum_{h, g \in S_{3}} \chi_{q}(h, g) B_{h}(s, p) A_{g}(s),
$$

where the characters $\chi_{q}$ associated with an irreducible representation $q$ can be calculated [31.

\subsection{Pinning Quasi-Particles}

We have not been able to fully characterise the spectrum of the Hamiltonian (7), with $A_{s}$ and $B_{p}$ defined as in Eq.430. However, we can argue that we actually do not need to as far as we are interested in the topological mutual information. Excited states of $H$ are tagged by vertices $s$ on the lattice that violate the condition $\left\langle A_{s}\right\rangle=1$ and plaquettes $p$ that violate the condition $\left\langle B_{p}\right\rangle=1$. We restrict to that part of the spectrum of $H$ such that excitations are pinned at fixed, non-adjacent locations (see Fig. 9 for a representation of a planar region). We expect that the more the noise undergone by the system, the less the topological mutual information. Considering only that restricted part of the spectrum of $H$ can be understood as additional error correction, where some plaquettes and vertices are over-protected so that they never get excited (or only with vanishing probability). It is actually easy to write a local Hamiltonian that automatically does that. Therefore, we believe that the topological mutual information of the modified model can only be larger than that of the full spectrum. 


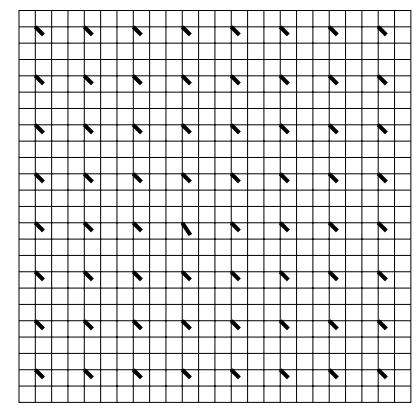

Figure 9: Square lattice associated with a quantum double model. Extra edges closing the lattice onto a sphere are not represented. Thick strokes refer to sites of the truncated model where excitations, living on a vertex and a plaquette (site), lie apart from each other.

We can also provide a renormalisation argument to support the idea of working with a truncated spectrum. Fixing the distance between two neighbour excitation sites to, say, two lattice spacings, and increasing the size of the lattice, i.e. the number of links, we see that the truncated model has a continuum limit, where excitations are pointwise anyonic particles (or superpositions thereof). We therefore expect that the potential differences between a situation where we consider the full spectrum and a situation where we consider only the truncated spectrum vanish in the limit of large lattices.

Our third argument is that in the case of the $\mathbb{Z}_{2}$ toric code, it does not make any difference whether we work with the full spectrum or with the truncated spectrum. This is most easily seen by considering the partition function of this model. Had we computed it for a system with more edges but still only $L^{2}$ possible positions for the charge (resp. flux) defects, we would have found exactly the same expression. Similarly, one easily checks that the topological mutual information remains unchanged.

\subsection{The fusion space and partition function}

The space of $n$ excitations pinned at fixed sites has the structure 3

$$
\mathcal{H}[n]=\bigoplus_{q_{1} \ldots q_{n}} \mathcal{H}_{q_{1} \ldots q_{n}},
$$

where each index $q_{i}$ runs over all possible quasiparticle types $A, \ldots, H$. This structure merely reflects the fact that different excitation patterns lead to orthogonal states. Each space $\mathcal{H}_{q_{1} \ldots q_{n}}$ further splits as

$$
\mathcal{H}_{q_{1} \ldots q_{n}}=\mathcal{K}_{q_{1}} \otimes \ldots \otimes \mathcal{K}_{q_{n}} \otimes \mathcal{M}_{q_{1} \ldots q_{n}} .
$$

The factor spaces $\mathcal{K}_{q_{i}}$ have a familiar meaning. They correspond to the local degrees of freedom of the excitations. The point of topological models is that the 
extra piece $\mathcal{M}_{q_{1} \ldots q_{n}}$, the fusion space, may have a non-trivial dimension. The particles can be fused. That is, two particles can be converted into one using a ribbon operator to connect them [3]. In the simple Abelian case discussed previously, the fusion rules were simple. For example, two plaquette excitations can only be fused in a way that makes them disappear. In a general anyonic model, the fusion rules read

$$
q_{a} \times q_{b}=\sum_{c} N_{a b}^{c} q_{c}
$$

where $N_{a b}^{c}$ are non-negative integers. We assume that physical states have to fulfill some neutrality conditions, i.e. they should be such that fusing all the particles yields with certainty the trivial particle, denoted as 1 . This assumption is legitimate since we work with a sphere [7. An important result of the theory of anyons is a formula for the dimension of $\mathcal{M}_{q_{1} \ldots q_{n}}$ [13]:

$$
\operatorname{dim} \mathcal{M}_{q_{1} \ldots q_{n}}=\sum_{b_{1}} \ldots \sum_{b_{n-2}} N_{q_{1} q_{2}}^{b_{1}} N_{b_{1} q_{3}}^{b_{2}} \ldots N_{b_{n-2} q_{n}}^{1} .
$$

This formula has a structure that makes it easy to compute for arbitrary $n$. $\operatorname{dim} \mathcal{M}_{q_{1} \ldots q_{n}}$ is the contraction of a (quasi) translationally invariant matrix product state [32. The dimension of $\mathcal{H}[n]$ can also be calculated easily. Let $d_{q}$ denote the dimension associated with the quasiparticle $q$. For $D\left(S_{3}\right)$ anyons, we have that $\left(d_{A}, d_{B}, d_{C}, d_{D}, d_{E}, d_{F}, d_{G}, d_{H}\right)=(1,1,2,3,3,2,2)$ [13. Defining the elements of a matrix $M$ as $M_{\alpha \beta}=\sum_{\gamma} N_{\alpha \gamma}^{\beta} d_{\gamma}, \operatorname{dim} \mathcal{H}[n]$ can be expressed as

$$
\operatorname{dim} \mathcal{H}[n]=\sum_{q_{1} \ldots q_{n}} \operatorname{dim} \mathcal{H}_{q_{1} \ldots q_{n}}=\sum_{\alpha} d_{\alpha} M_{\alpha, 1}^{n-1}
$$

The partition function of the model on a sphere, with $n$ well separated sites reads

$$
Z(\beta, n)=\sum_{q_{1}} \ldots \sum_{q_{n}} e^{-\beta E\left(q_{1} \ldots q_{n}\right)} d_{q_{1}} \ldots d_{q_{n}} N_{q_{1} q_{2}}^{b_{1}} N_{b_{1} q_{3}}^{b_{2}} \ldots N_{b_{n-2} q_{n}}^{1},
$$

where $E\left(q_{1} \ldots q_{n}\right)$ is the energy associated with a configuration $q_{1} \ldots q_{n}$. Just like $\operatorname{dim} \mathcal{H}[n]$, it can be computed efficiently upon diagonalizing a matrix $M$, whose size is independent of $n$.

\section{4 von Neumann entropy at finite temperature}

Let us start by considering a scenario where a pair of anyons $|q \bar{q}\rangle$ is created in such a way that anyon $q$ lies in some region $A$ and anyon $\bar{q}$ lies in the complementary region. In this configuration, the von Neumann entropy of region $A$ reads [21]

$$
S_{q}^{\text {pair }}\left(\rho_{A}\right)=S\left(\rho_{A}^{\text {g.s. }}\right)+\log d_{q} .
$$


The entropy of a region when the system is in a thermal state can be computed once we are able to calculate the entropy of a region when the system is an arbitrary defect configuration. In turn, the latter entropy reduces to computing the entropy when there are only two anyons in the system, and one lies inside the region we are interested in.

Consider a tiled sphere as the one indicated in Fig. 8, and let us divide it into two simply connected regions, $A$ and $B$. Let $N_{A}$ and $N_{B}$ denote the number of sites contained in region $A$ and $B$ respectively $\left(N_{A}+N_{B} \equiv n\right)$. Let $\mathbf{q}_{A}=q_{1} \ldots q_{N_{A}}$ label the types of anyons living on the sites contained in region $A$ and $\mathbf{q}_{B}^{\prime}=q_{1}^{\prime} \ldots q_{B}^{\prime}$ label the types of anyons living on the sites contained in region $B$. The total Hilbert space can be decomposed as:

$$
\mathcal{H}[n]=\bigoplus_{\mathbf{q}_{A}} \bigoplus_{\mathbf{q}_{B}^{\prime}} \mathcal{K}_{\mathbf{q}_{A}} \otimes \mathcal{K}_{\mathbf{q}_{B}^{\prime}} \otimes \bigoplus_{b} \mathcal{M}_{\mathbf{q}_{A}}^{b} \otimes \mathcal{M}_{\mathbf{q}_{B}^{\prime}}^{\bar{b}} \otimes \mathcal{M}_{b, \bar{b}}^{1}
$$

(N.B. The spaces $\mathcal{M}_{b, \bar{b}}^{1}$ are one-dimensional.) The decomposition $\sqrt{52}$ induces the following representation of the thermal state of the $D\left(S_{3}\right)$ superconductor

$$
\begin{aligned}
& \rho_{\mathrm{th}}=\bigoplus_{\mathbf{q}_{A}} \bigoplus_{\mathbf{q}_{B}^{\prime}} \bigoplus_{b=A}^{H} \bigoplus_{\mu_{1}=1}^{\operatorname{dim} \mathcal{M}_{\mathbf{q}_{A}}^{b}} \bigoplus_{\mu_{2}=1}^{\operatorname{dim} \mathcal{M}_{\mathbf{q}_{B}^{\prime}}^{\bar{b}}} \bigoplus_{s_{A}=1}^{d\left(\mathbf{q}_{A}\right)} \bigoplus_{s_{B}=1}^{d\left(\mathbf{q}_{B}^{\prime}\right)} \frac{e^{-\beta\left(E\left(\mathbf{q}_{A}\right)+E\left(\mathbf{q}_{B}^{\prime}\right)\right)}}{Z(\beta, n)} \\
& \left|\mathbf{q}_{A} \rightarrow b, \mu_{1}, s_{A} ; \mathbf{q}_{B}^{\prime} \rightarrow \bar{b}, \mu_{2}, s_{B}\right\rangle\left\langle\mathbf{q}_{A} \rightarrow b, \mu_{1}, s_{A} ; \mathbf{q}_{B}^{\prime} \rightarrow \bar{b}, \mu_{2}, s_{B}\right|,
\end{aligned}
$$

where $E\left(\mathbf{q}_{A}\right)=\sum_{j=1}^{N_{A}} E\left(q_{j}\right), d\left(\mathbf{q}_{A}\right)=\prod_{j=1}^{N_{A}} d\left(q_{j}\right), \mu_{1}$ (resp. $\left.\mu_{2}\right)$ denotes the possible channels through which the anyons $\mathbf{q}_{A}$ (resp. $\mathbf{q}_{B}{ }_{B}$ ) can be fused into $b$ (resp. $\bar{b}$ ), and $s_{A}$ (resp. $s_{B}$ ) is a collective index for the internal degrees of freedom of the quasiparticles contained in region $A$ (resp. $B$ ).

Defining

$$
Z_{A}(\beta, b) \equiv \sum_{\mathbf{q}_{A}} d\left(\mathbf{q}_{A}\right) e^{-\beta E\left(\mathbf{q}_{A}\right)} \operatorname{dim} \mathcal{M}_{\mathbf{q}_{A}}^{b},
$$

and $Z_{B}(\beta, \bar{b})$ likewise, we get that the reduced state of region $A$ reads

$\rho_{A}=\bigoplus_{b, \mathbf{q}_{A}} \bigoplus_{\mu_{1}=1}^{\operatorname{dim} \mathcal{M}_{\mathbf{q}_{A}}^{b}} \bigoplus_{s_{A}=1}^{d\left(\mathbf{q}_{A}\right)} \frac{e^{-\beta E\left(\mathbf{q}_{A}\right)} Z_{B}(\beta, \bar{b})}{Z(\beta, n)} \operatorname{tr}_{B}\left|\mathbf{q}_{A} \rightarrow b, \mu_{1}, s_{A} ; \bar{b}\right\rangle\left\langle\mathbf{q}_{A} \rightarrow b, \mu_{1}, s_{A} ; \bar{b}\right|$

It is worth observing the resemblance between Eq. (54) and the reduced density matrix for the toric code. From Eq. (54) and Eq. $(20)$, we deduce an expression 
for the entropy of system $A$ :

$$
S\left(\rho_{A}\right)=-\sum_{\mathbf{q}_{A}, b, \mu_{1}, s_{A}} \frac{e^{-\beta E\left(\mathbf{q}_{A}\right)} Z_{B}(\beta, \bar{b})}{Z(\beta, n)}\left\{\ln \left[\frac{e^{-\beta E\left(\mathbf{q}_{A}\right)} Z_{B}(\beta, \bar{b})}{Z(\beta, n)}\right]-S_{q}^{\text {pair }}\left(\rho_{A}\right)\right\}
$$

Just as for the partition function, $S\left(\rho_{A}\right)$ can be computed by diagonalization of an auxiliary matrix. Finally, the von Neumann entropy of the whole sphere can be efficiently computed from the partition function using again Eq. 15.)

These expressions are valid for any quantum double model on a lattice such as the one depicted in Fig. 9. Remarkably, the only microscopic data involved in the expressions for $S\left(\rho_{A}\right)$ and $S\left(\rho_{\mathrm{th}}\right)$ are the tensor $N$ governing the fusion rules, the energies associated with each quasiparticle type and their internal dimension. It is therefore tempting to conjecture that these expressions are valid for other anyonic theories.

The fact that the fusion rules tensor $N_{* *}^{*}$ appears in the expression for the finite temperature entropy of a region marks a qualitative difference with respect to the zero temperature (ground state) case. This is interesting in that it shows that entropic quantities could be used in order to discriminate between different topological models. For example, at finite temperature, the quantum double models based on $\mathbb{Z}_{6}$ and $S_{3}$ exhibit different region entropies, while these quantities coincide at zero temperature. We understand this difference as follows: at finite temperature, quasiparticle pairs can be spontaneously created from the vacuum and can move around on the lattice. When they braid, they give rise to qualitatively different statistics, which in turn give rise to different values for entropic quantities.

\subsection{Numerical Results}

The results derived in the previous section, and a rigorous calculation of the ground state entropy, shown in Appendix Chave allowed us to study numerically how the topological mutual information behaves as a function of $\beta$. The systems we have considered are four tiled spheres, all with $96 \times 96$ plaquettes. The first sphere contains 64 sites, the second 144, the third 256 and the fourth 576 . Although these systems are small when compared to their Abelian counterpart we have considered on Fig 4, they are large enough to show that non-Abelian systems are affected by temperature in the same way as the toric code [12].

Fig. 10 is presented to analyze the existence of scaling laws in the nonAbelian case. There, the topological mutual information is represented as a function of $n \exp (-2 \beta)$. We can see that the curves tend to collapse when the number of sites is increased, that is, the distance between the four curves reduces. This fact supports the idea that for large systems, the topological mutual information only depends on the 'volume' of the system and the temperature through the product $n \exp (-2 \beta)$. This result is consistent with the scaling relation found for the toric code. 


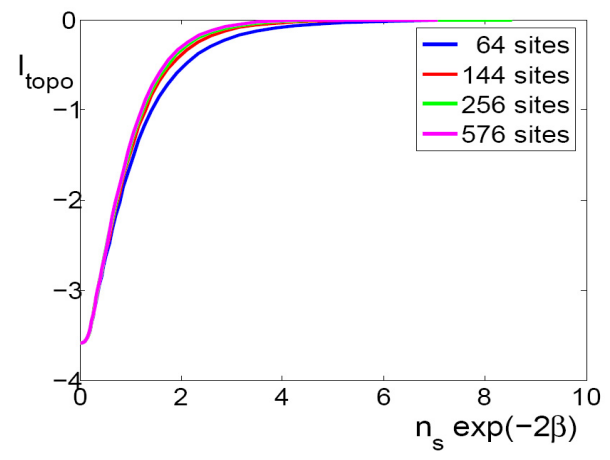

Figure 10: Topological mutual information as a function of $n \exp (-2 \beta)$ for the $D\left(S_{3}\right)$ model.

\section{Conclusions}

In conclusion, we have studied how topological phases of lattice systems behave as a function of temperature, using the topological mutual information as an order parameter. We have focused on two specific models; the toric code and the $D\left(S_{3}\right)$ quantum double model. These systems turned out to be affected by temperature in much the same way. We have studied how the topological mutual information depends on size and temperature through a simple scaling variable. In particular, we have shown how an increase of system size can be compensated by a vanishing decrease of temperature. It would be interesting to see whether our findings are also valid for other systems, such as a FQHE sample for instance. We have also seen how temperature could allow to resolve between models whose topological entropy are equal at zero temperature. We have also introduced a class of simulations where running time simulates increasing temperature. Though exemplified by a minimal plaquette in the Abelian model, it can be extended to more complicated situations. We have also discussed why, at finite temperature, $I_{\text {topo }}$ should be used as an appropriate topological order parameter instead of the topological entropy. We have shown how $I_{\text {topo }}$ relates directly to the thermal probability distribution of topological charges, and how in turn this distribution is related to the mean value of $S$-matrix elements and controls the visibility of anyonic processes, and therefore the usefulness of topologically ordered systems as quantum memories.

\section{Acknowledgements}

We thank Gavin Brennen, Ignacio Cirac, José Ignacio Latorre, Miguel-Angel Martin-Delgado, Ady Stern and Paolo Zanardi for inspiring discussions. We also thank Miguel-Angel Martin-Delgado for having given us an early version of 
Ref. [7. Financial support from the Generalitat de Catalunya, MEC (Spain), QAP, EMALI, SCALA (EU), Spanish grants MTM2005-00082, I-MATH, and CCG07-UCM/ESP-2797, and UK grants from EPSRC and the Royal Society.

\section{A Some calculations for the toric code}

Most of the calculations presented in this appendix are based on repeated use of three elementary identities:

$$
\begin{aligned}
& \sum_{k=0}^{N}\left(\begin{array}{l}
N \\
k
\end{array}\right) \alpha^{k}=(1+\alpha)^{N}, \\
& \sum_{\substack{k=0 \\
k \text { even }}}^{N}\left(\begin{array}{l}
N \\
k
\end{array}\right) \alpha^{k}=\frac{1}{2} \sum_{k=0}^{N}\left(\begin{array}{l}
N \\
k
\end{array}\right)\left(\alpha^{k}+(-\alpha)^{k}\right), \\
& \sum_{k=0}^{N}\left(\begin{array}{l}
N \\
k
\end{array}\right) k \alpha^{k}=\alpha \frac{\partial}{\partial \alpha}(1+\alpha)^{N}=N \alpha(1+\alpha)^{N-1} .
\end{aligned}
$$

One finds that the partition function reads:

$$
\begin{aligned}
Z(\beta, L) & =\sum_{i=0}^{L^{2}} e^{-\beta E_{i}} d_{i}=\sum_{n_{\phi}=0}^{\left\lfloor L^{2} / 2\right\rfloor} \sum_{n_{c}=0}^{\left\lfloor L^{2} / 2\right\rfloor} e^{-\beta\left(E_{0}+4 n_{\phi}+4 n_{c}\right)}\left(\begin{array}{c}
L^{2} \\
2 n_{\phi}
\end{array}\right)\left(\begin{array}{c}
L^{2} \\
2 n_{c}
\end{array}\right) \\
& =\left[(2 \cosh \beta)^{L^{2}}+(2 \sinh \beta)^{L^{2}}\right]^{2}
\end{aligned}
$$

as derived in [15, 14.

Next we want to compute the quantity

$$
W(\beta, R, L) \equiv-\sum_{\mathbf{q}_{R}} 4 C\left(\mathbf{q}_{R}\right) \ln \left(4 C\left(\mathbf{q}_{R}\right)\right),
$$

appearing in 21). For that, it is convenient to consider plaquette and vertex excitations separately: $\mathbf{q}_{R} \equiv\left(\phi_{R}, c_{R}\right)$. Let $N_{p}(R)$ (resp. $\left.N_{*}(R)\right)$ denote the number of plaquette (resp. vertex) excitations whose edges are all contained in $R$, and let us define $N_{p}^{\prime}(R) \equiv L^{2}-N_{p}(R)$ and $N_{*}^{\prime}(R)=L^{2}-N_{*}(R)$. With these notations, $C\left(\mathbf{q}_{R}\right)$ can be decomposed as

$$
C\left(\mathbf{q}_{R}\right)=\frac{e^{-\beta\left(E_{0}+2\left|\phi_{R}\right|+2\left|c_{R}\right|\right)}}{Z(\beta, L)} \mathcal{P}\left(\left|\phi_{R}\right|, N_{p}^{\prime}(R), e^{-2 \beta}\right) \mathcal{P}\left(\left|c_{R}\right|, N_{*}^{\prime}(R), e^{-2 \beta}\right),
$$

where

$$
\mathcal{P}(m, N, \alpha)=\sum_{\substack{n=0 \\
n+m \text { even }}}^{N}\left(\begin{array}{l}
N \\
n
\end{array}\right) \alpha^{n}=\frac{1}{2}\left\{(1+\alpha)^{N}+(-1)^{m}(1-\alpha)^{N}\right\} .
$$


In order to get an anlytical expression for $W(\beta, R, L)$, it is sufficient to evaluate sums like:

$$
S_{1}(R)=\sum_{\phi_{R}} e^{-2 \beta\left|\phi_{R}\right|} \mathcal{P}\left(\left|\phi_{R}\right|, N_{p}^{\prime}(R), e^{-2 \beta}\right),
$$

and

$$
\begin{aligned}
S_{2}(R) & =\sum_{\phi_{R}} e^{-2 \beta\left|\phi_{R}\right|} \mathcal{P}\left(\left|\phi_{R}\right|, N_{p}^{\prime}(R), e^{-2 \beta}\right) \ln \mathcal{P}\left(\left|\phi_{R}\right|, N_{p}^{\prime}(R), e^{-2 \beta}\right) \\
S_{1}(R) & =\sum_{\phi_{R}} e^{-2 \beta\left|\phi_{R}\right|} \mathcal{P}\left(\left|\phi_{R}\right|, N_{p}^{\prime}(R), e^{-2 \beta}\right) \\
& =\frac{1}{2} \sum_{m=0}^{N_{p}(R)} e^{-2 \beta m}\left(\begin{array}{c}
N_{p}(R) \\
m
\end{array}\right)\left\{\left(1+e^{-2 \beta}\right)^{N_{p}^{\prime}(R)}+(-)^{m}\left(1-e^{-2 \beta}\right)^{N_{p}^{\prime}(R)}\right\} \\
& =\frac{1}{2}\left\{\left(1+e^{-2 \beta}\right)^{L^{2}}+\left(1-e^{-2 \beta}\right)^{L^{2}}\right\}
\end{aligned}
$$

Splitting $S_{2}(R)$ as a sum over even values of $\left|\phi_{R}\right|$ and a sum over odd values of $\left|\phi_{R}\right|$, we get that

$$
\begin{aligned}
S_{2}(R) & =\frac{1}{4}\left\{\left(1+e^{-2 \beta}\right)^{N_{p}(R)}+\left(1-e^{-2 \beta}\right)^{N_{p}(R)}\right\}\left\{\left(1+e^{-2 \beta}\right)^{N_{p}^{\prime}(R)}+\left(1-e^{-2 \beta}\right)^{N_{p}^{\prime}(R)}\right\} \\
& \ln \left[\frac{1}{2}\left\{\left(1+e^{-2 \beta}\right)^{N_{p}^{\prime}(R)}+\left(1-e^{-2 \beta}\right)^{N_{p}^{\prime}(R)}\right\}\right] \\
+ & \frac{1}{4}\left\{\left(1+e^{-2 \beta}\right)^{N_{p}(R)}-\left(1-e^{-2 \beta}\right)^{N_{p}(R)}\right\}\left\{\left(1+e^{-2 \beta}\right)^{N_{p}^{\prime}(R)}-\left(1-e^{-2 \beta}\right)^{N_{p}^{\prime}(R)}\right\} \\
& \ln \left[\frac{1}{2}\left\{\left(1+e^{-2 \beta}\right)^{N_{p}^{\prime}(R)}-\left(1-e^{-2 \beta}\right)^{N_{p}^{\prime}(R)}\right\}\right]
\end{aligned}
$$

With these results, we find Eqs.22, 23.

\section{B Rough edges and smooth edges}

When dividing a lattice into four regions as indicated in Fig. 1, one has to be careful that the links on the boundary between two regions should be attributed only to one of them. On Fig 11 , we show how we have chosen to divide Fig.1 Data related to the division are collected in Table 1.

In the Abelian case, the entropy of a region $R$ in a zero temperature thermal state (i.e. in an equal mixture of four basis states of the ground states) is given by $\left(\left|\partial_{R}\right|-1\right) \ln 2-\epsilon_{R}$. The $\epsilon_{R}$ correction appears because we have worked with a model defined on a torus, and takes into account the fact that the region $R$ might be contractible. The "area" $|\partial R|$ is given by the total number of crosses 
minus the number of crosses fully inside $R$ minus the number of crosses fully outside $R$.

The non-Abelian $D\left(S_{3}\right)$ case has been studied on a sphere. There the ground state entropy is given by $\left(\left|\partial_{R}\right|-1\right) \ln 6$, see Appendix C. As shown in Fig. 8 , there is a region within the sphere made of square plaquettes only. (We have chosen our tiling of the sphere in this way.) Let $L$ denote the (linear) size of this region, and $k$ denotes the linear size of region $A$. (All triangular plaquettes are assumed to be inside region $D$.) In order to ease the computations, we choose $L$ to be a multiple of $k$, and we impose that the density of sites, $s^{2}$, is such that $s^{-1}$ is a divisor of $k$. (Triangular plaquettes are assumed to support no site.)
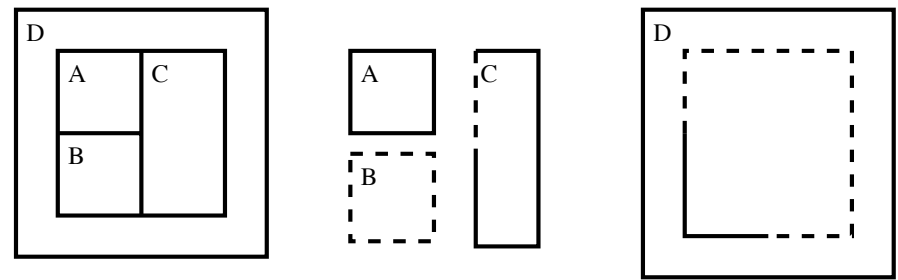

Figure 11: Division of the lattice into four regions used in numerical calculations. Delimitations of a region by dotted lines indicate that the corresponding links do not belong to the region.

\begin{tabular}{|c|c|c|c|c|c|}
\hline Region & "Area"-1 & $\epsilon$ & $N_{p}$ & $N_{*}$ & "Volume" \\
\hline $\mathrm{A}$ & $4 k-1$ & $-\log 4$ & $k^{2}$ & $(k-1)^{2}$ & $k^{2}$ \\
$\mathrm{~B}$ & $4 k-5$ & $-\log 4$ & $k^{2}-4 k+4$ & $(k-1)^{2}$ & $k^{2}$ \\
$\mathrm{C}$ & $6 k-1$ & $-\log 4$ & $2 k^{2}-k$ & $(k-1)(2 k-1)$ & $2 k^{2}$ \\
$\mathrm{D}$ & $8 k-2$ & 0 & $L^{2}-4 k^{2}-6 k$ & $L^{2}-4 k^{2}-4 k$ & $L^{2}-4 k^{2}$ \\
$\mathrm{AB}$ & $6 k-3$ & $-\log 4$ & $2 k^{2}-3 k+2$ & $(k-1)(2 k-1)$ & $2 k^{2}$ \\
$\mathrm{AC}$ & $8 k-2$ & $-\log 4$ & $3 k^{2}$ & $3 k^{2}-4 k+2$ & $3 k^{2}$ \\
$\mathrm{AD}$ & $8 k-3$ & 0 & $L^{2}-3 k^{2}-4 k$ & $L^{2}-3 k^{2}-4 k+1$ & $L^{2}-3 k^{2}$ \\
$\mathrm{BC}$ & $8 k-3$ & $-\log 4$ & $3 k^{2}-4 k+2$ & $3 k^{2}-4 k+1$ & $3 k^{2}$ \\
$\mathrm{BD}$ & $8 k-2$ & 0 & $L^{2}-3 k^{2}-8 k+1$ & $L^{2}-3 k^{2}-4 k-1$ & $L^{2}-3 k^{2}$ \\
$\mathrm{CD}$ & $6 k-3$ & 0 & $L^{2}-2 k^{2}-3 k$ & $L^{2}-2 k^{2}-3 k+1$ & $L^{2}-2 k^{2}$ \\
$\mathrm{ABC}$ & $8 k-2$ & $-\log 4$ & $4 k^{2}-2 k+1$ & $(2 k-1)^{2}$ & $4 k^{2}$ \\
$\mathrm{ABD}$ & $6 k-1$ & 0 & $L^{2}-2 k^{2}-5 k$ & $L^{2}-2 k^{2}-3 k-1$ & $L^{2}-2 k^{2}$ \\
$\mathrm{ACD}$ & $4 k-5$ & 0 & $L^{2}-k^{2}$ & $L^{2}-k^{2}-2 k+3$ & $L^{2}-k^{2}$ \\
$\mathrm{BCD}$ & $4 k-1$ & 0 & $L^{2}-k^{2}-4 k$ & $L^{2}-k^{2}-2 k-1$ & $L^{2}-k^{2}$ \\
\hline
\end{tabular}

Table 1: Some data useful to compute the von Neumann entropies of various regions of a tiled torus at finite temperature. The regions are those depicted in Fig. 11. $\epsilon$ labels the correction to the ground state entropy due to the topology of the considered region. $N_{p}$ (resp. $N_{*}$ ) denotes the number of plaquettes (resp. crosses) fully contained in the region. 


\section{Ground State Entanglement of the $D\left(S_{3}\right)$ su- perconductor}

Here, we generalize some of the calculations presented in 33 and derive a simple exact expression for the entropy of the reduced matrix $\rho_{A}$ of the ground state $|\xi\rangle$ in a region $A$. ( $B$ here denotes the complementary of $A$ ). We need to distinguish amongst three types of vertices, those in $A$, those in $B$ and those touched by edges belonging to $A$ and edges belonging to $B$. The set of vertices of the latter kind will be referred to as $\partial A$. We consider only regions with the following properties

1. Both $A$ and $B$ are connected in the sense of vertices. That is, every two vertices inside $A$ (resp. $B$ ) can be connected by a path of adjacent vertices inside $A$ (resp. $B$ ).

2. For each $s \in \partial A$, there exist $s^{\prime} \in A, s^{\prime \prime} \in B$ adjacent to $s$.

The (unnormalized) ground state of the $D\left(S_{3}\right)$ model defined on a sphere can be written as

$$
|\xi\rangle=\sum_{g_{1}, \ldots, g_{N}} A_{g_{1}}\left(s_{1}\right) \cdots A_{g_{N}}\left(s_{N}\right)|e \cdots e\rangle=\sum_{\mathbf{g} \in \mathbf{G}} \pi(\mathbf{g})|e \cdots e\rangle,
$$

where $\mathbf{g} \equiv\left(g_{1}, \ldots, g_{N}\right), \mathbf{G}=S_{3} \times \cdots \times S_{3}$ and $\pi$ is the representation of $\mathbf{G}$ defined by $\pi(\mathbf{g})=A_{g_{1}}\left(s_{1}\right) \cdots A_{g_{N}}\left(s_{N}\right)$. The action of $\pi(\mathbf{g})$ can be decomposed as a product of unitaries acting on the edges of the lattice:

$$
\pi(\mathbf{g})=\bigotimes_{\left\langle s_{i}, s_{j}\right\rangle} U_{\left\langle s_{i}, s_{j}\right\rangle}\left(g_{i}, g_{j}\right)
$$

where the operator $U_{\left\langle s_{i}, s_{j}\right\rangle}$ acts as follows on the 'computational' basis states $\left\{|x\rangle: x \in S_{3}\right\}$ of the edge $\left\langle s_{i}, s_{j}\right\rangle: U_{\left\langle s_{i}, s_{j}\right\rangle}|x\rangle=\left|g_{i} x g_{j}^{-1}\right\rangle$ if the edge goes from the vertex $s_{i}$ to $s_{j}$. Therefore $\left.\left.U_{\left\langle s_{i}, s_{j}\right\rangle}\right\rangle g_{i}, g_{j}\right)$ acts trivially on an edge $\left\langle s_{i}, s_{j}\right\rangle$ only if $g_{i}$ equals $g_{j}$ and belongs to the centraliser of $S_{3}$, which is $\{e\}$. Therefore, the representation $\pi$ is faithful and we can (and will) identify $\mathbf{G}$ with $\pi(\mathbf{G})$.

We now borrow some definitions from [33. We define two normal subgroups in $\mathbf{G}$ :

$$
\begin{aligned}
\mathbf{G}_{A} & =\left\{\mathbf{g} \in \mathbf{G} \mid g_{j}=e \text { if } s_{j} \notin A\right\}, \\
\mathbf{G}_{B} & =\left\{\mathbf{g} \in \mathbf{G} \mid g_{j}=e \text { if } s_{j} \notin B\right\},
\end{aligned}
$$

and we call $\mathbf{G}_{A B}$ the quotient group $\mathbf{G} /\left(\mathbf{G}_{A} \times \mathbf{G}_{B}\right)$.

Any element of $\mathbf{G}_{X}(X=A, B)$ acts only on $X$ and we have the following partition of $\mathbf{G}$ :

$$
\mathbf{G}=\cup_{[\mathbf{h}] \in \mathbf{G}_{A B}}\left\{\left(\mathbf{g}_{A} \otimes \mathbf{g}_{B}\right) \mathbf{h} \mid \mathbf{g}_{A} \in \mathbf{G}_{A}, \mathbf{g}_{b} \in \mathbf{G}_{B}\right\},
$$


where we can fix any representative $\mathbf{h}$ for the class $[\mathbf{h}]$. This decomposition implies that the ground state can be expressed as

$$
|\xi\rangle=Q_{A} \otimes Q_{B} \sum_{[\mathbf{h}]} \mathbf{h}|e \cdots e\rangle,
$$

where $Q_{X}=\sum_{\mathbf{g}_{X} \in \mathbf{G}_{X}} \mathbf{g}_{X}$.

For our purposes, we need to quotient further $G_{A B}$. So, we consider the diagonal of $\mathbf{G}, \mathbf{G}_{\mathrm{d}}=\left\{\tilde{r}=(r, \cdots, r) \mid r \in S_{3}\right\}$, and the quotient set, $\mathbf{G}_{A B} / \mathbf{G}_{\mathrm{d}}$, defined by the (right) equivalence relation: $[\mathbf{h}] \sim\left[\mathbf{h}^{\prime}\right]$ iff $\exists r \in S_{3}$ s.t. $[\mathbf{h}] \sim\left[\mathbf{h}^{\prime} \tilde{r}\right]$. Clearly, $\left|\mathbf{G}_{A B} / \mathbf{G}_{\mathrm{d}}\right|=\left|\mathbf{G}_{A B}\right| /\left|S_{3}\right|$. We denote [[h]] the elements of $\mathbf{G}_{A B} / \mathbf{G}_{\mathrm{d}}$. Since $\mathbf{G}_{\mathrm{d}}$ acts trivially on $|e \ldots e\rangle,|\xi\rangle$ can be further decomposed as

$$
|\xi\rangle=Q_{A} \otimes Q_{B} \sum_{r \in S_{3}} \sum_{[[\mathbf{h}]]} \mathbf{h} \tilde{r}|e \ldots e\rangle=\left|S_{3}\right| Q_{A} \otimes Q_{B} \sum_{[[\mathbf{h}]]} \mathbf{h}_{A} \otimes \mathbf{h}_{B}|e \ldots e\rangle,
$$

with $\mathbf{h}=\mathbf{h}_{A} \otimes \mathbf{h}_{B}$, and where $\mathbf{h}_{A}$ (resp. $\mathbf{h}_{B}$ ) does not necessarily belong to $\mathbf{G}_{A}$ $\left(\right.$ resp. $\left.\mathbf{G}_{B}\right)$. In order to conclude our calculation of $S\left(\rho_{A}\right)$, we use the following lemma.

Lemma $\mathbf{1}$ Let $\mathbf{g}$ denote an arbitrary element of $\mathbf{G}$ and let $\mathbf{g}=\mathbf{g}_{A} \otimes \mathbf{g}_{B}$ denote its decomposition into an operator acting on $A$ and an operator acting on $B$. If for all $r \in S_{3},[\mathbf{g}] \neq[\tilde{r}]$, then $\left\langle e \ldots e\left|\mathbf{g}_{X}\right| e \ldots e\right\rangle=0$ for both $X=A, B$.

Proof: Let us suppose that it is false. $[\mathbf{g}] \neq[\tilde{r}]$ iff there are at least two vertices $s_{i}, s_{j} \in \partial A$ such that $g_{i} \neq g_{j}$. Let us consider a path $\Gamma_{X}$ connecting $s_{i}$ to $s_{j}$ through edges within $X$. Since

$$
\left\langle e \ldots e\left|\mathbf{g}_{X}\right| e \ldots e\right\rangle=\prod_{\left\langle s_{\alpha}, s_{\beta\rangle} \in X\right.}\left\langle e\left|g_{\alpha}^{-1} g_{\beta}\right| e\right\rangle,
$$

we have that $\left\langle e \ldots e\left|\mathbf{g}_{X}\right| e \ldots e\right\rangle \neq 0$ only if $g_{\alpha}=g_{\beta}$ for all pairs of adjacent vertices $s_{\alpha}, s_{\beta}$. Thus $\left\langle e \ldots e\left|\mathbf{g}_{X}\right| e \ldots e\right\rangle \neq 0$ only if $g_{i}=g_{\alpha}$ for all vertex $s_{\alpha} \in$ $\Gamma_{X}$, and in particular $g_{i}=g_{j}$, which is the desired contradiction.

This lemma allows us to prove that the states $\left\{\left(\mathbf{h}_{A} \otimes \mathbf{h}_{B}\right)|e \ldots e\rangle:[[\mathbf{h}]] \in\right.$ $\left.\mathbf{G}_{A B} / \mathbf{G}_{\mathrm{d}}\right\}$ form a bi-orthogonal set, so that the expression 62 is actually a Schmidt decomposition of the ground state. If $[[\mathbf{h}]]$ and $\left[\left[\mathbf{h}^{\prime}\right]\right]$ are different equivalence classes, then $\left\langle e \ldots e\left|\mathbf{h}_{X}^{-1} \mathbf{h}_{X}^{\prime}\right| e \ldots e\right\rangle=0$ for both $X=A, B$. Indeed, $[[\mathbf{h}]] \neq\left[\left[\mathbf{h}^{\prime}\right]\right]$ means that $[\mathbf{h}] \neq\left[\mathbf{h}^{\prime} \tilde{r}\right]$ for all $r \in S_{3}$. That is $\left[\mathbf{h}^{-1} \mathbf{h}^{\prime}\right] \neq[\tilde{r}]$ and the lemma 1 shows that the desired property holds. At this point, the construction of Ref. [33] can be used to first prove that the set $\left\{\left(Q_{A} \mathbf{h}_{A} \otimes Q_{B} \mathbf{h}_{B}\right)|e \ldots e\rangle\right.$ : $\left.[[\mathbf{h}]] \in \mathbf{G}_{A B} / \mathbf{G}_{d}\right\}$ is also a bi-orthogonal set, and then on to show that

$$
S\left(\rho_{A}\right)=\log _{2} \frac{\left|\mathbf{G}_{A B}\right|}{\left|S_{3}\right|}=\log _{2}\left|S_{3}\right|\left(N_{\partial A}-1\right),
$$


where $N_{\partial A}$ is the number of vertices on the boundary of $A$.

Remark: The argument just presented for $S_{3}$ is actually valid for any finite group $G$. If $G$ has a non-trivial centre $Z(G) \neq\{e\}$, then one would merely start the construction from the group $\mathbf{G} / Z(\mathbf{G})_{\mathrm{d}}$ instead of $\mathbf{G}$.

\section{References}

[1] N. W. Ashcroft and N. D. Mermin, Solid State Physics, Brooks-Cole (1976).

[2] D. Yoshioka, The Quantum Hall Effect, Springer (2002). Z. F. Ezawa, Quantum Hall Effects, Field Theoretical Approach and Related Topics, World Scientific (2000).

[3] A. Yu. Kitaev, Annals Phys. 303, 2 (2003).

[4] S. Das Sarma, M. Freedman, C. Nayak, S. H. Simon and A. Stern, e-print arXiv:0707.1889

[5] M. Levin and X.-G. Wen, Phys. Rev. B 71, 045110 (2005).

[6] B. Douçot, L. B. Ioffe and J. Vidal, Phys. Rev. B 69, 214501 (2004).

[7] H. Bombín and M. Á. Martín-Delgado, Phys. Rev. B 78, 115421 (2008).

[8] E. Dennis, A. Kitaev, A. Landahl and J. Preskill, J. Math. Phys. 43, 4452 (2002).

[9] C. Wang, J. Harrington and J. Preskill, Annals Phys. 303, 31 (2003).

[10] C. Mochon, Phys. Rev. A 69, 032306 (2004).

[11] G. K. Brennen and J. K. Pachos, Proc. Roy. Soc. A 464, 1 (2007).

[12] S. Iblisdir, D. Perez-García, M. Aguado, J. Pachos, e-print arXiv:0806.1853, to appear in Phys. Rev. B.

[13] J. Preskill, Lectures notes on Quantum Computation, Chap. 9, www.theory.caltech.edu/people/preskill/ph229/.

[14] Z. Nussinov and G. Ortiz, e-print arXiv:cond-mat/0702377.

[15] C. Castelnovo and C. Chamon, Phys. Rev. B 76, 184442 (2007).

[16] A. Kay, e-print arXiv:0807.0287.

[17] R. Alicki, M. Horodecki, P. Horodecki, R. Horodecki, e-print arXiv:0811.0033

[18] A. Kay, R. Colbeck, e-print arXiv:0810.3557.

[19] S. Bravyi, B. Terhal, e-print arXiv:0810.1983. 
[20] D. F. Abasto and P. Zanardi, e-print arXiv:0809.4740.

[21] A. Kitaev and J. Preskill, Phys. Rev. Lett. 96, 110404 (2006).

[22] M. Levin and X.-G. Wen, Phys. Rev. Lett. 96, 110405 (2006).

[23] A. Hamma, R. Ionicioiu, P. Zanardi, Phys. Lett. A 337, 22 (2005).

[24] M. M. Wolf, F. Verstraete, M. B. Hastings and J. I. Cirac, Phys. Rev. Lett. 100, 070502 (2008).

[25] J. K. Pachos, W. Wieczorek, C. Schmid, N. Kiesel, R. Pohlner, H. Weinfurter, e-print arXiv:0710.0895. J.-F. Du, J. Zhu, M.-G. Hu and J.-L. Chen, e-print arXiv:0712.2694.

[26] M. Aguado, G. K. Brennen, F. Verstraete, J. I. Cirac, e-print arXiv:0802.3163, to appear in Phys. Rev. Lett.

[27] T. M. Cover and J. A. Thomas, Elements of Information Theory, John Wiley and Sons (1991).

[28] E. Fradkin, C. Nayak, A. Tsvelik and F. Wilczek, Nucl. Phys. B 516 (1998) 704. B. J. Overbosch, F. A. Bais, Phys. Rev. A 64 (2001) 062107. P. Bonderson, A. Kitaev, K. Shtengel, Phys. Rev. Lett. 96, 016803 (2006). P. Bonderson, K. Shtengel and J. K. Slingerland, Phys. Rev. Lett. 97, 016401 (2006).

[29] M. de Wild Propitius and F. A. Bais, e-print arXiv:hep-th/9511201.

[30] R. Dijkgraaf, V. Pasquier and P. Roche, Nucl. Phys. B (Proc. Suppl.) 18B (1990).

[31] B. J. Overbosch, The entanglement and measurement of non Abelian anyons as an approach to quantum computation, M. Sc. Thesis, University of Amsterdam (2000).

[32] S. Ostlund and S. Rommer, Phys. Rev. Lett. 75, 3537 (1995). M. Fannes, B. Nachtergaele, and R. F. Werner, Comm. Math. Phys. 144 (1992). D. Perez-García, F. Verstraete, M. M. Wolf, J. I. Cirac, Quantum Inf. Comput. 7, 401 (2007).

[33] A. Hamma, R. Ionicioiu and P. Zanardi, Phys. Rev. A 72, 012324 (2005). 